\title{
Negative effects of part-set cues: Are they reversible?
}

\author{
HEEKYEONG PARK and STEPHEN MADIGAN \\ University of Southern California, Los Angeles, California
}

(Joseph Hellige, Sponsor)

\begin{abstract}
In a number of studies, it has been reported that the negative effect of part-set cues on recall disappears when a second recall test is administered without cues. The present experiments show that the removal of cues does not significantly increase the recall of new items on a second test over that obtained in two successive tests without cues, or in two successive tests with cues. The part-set cuing effect does not appear to be reversible by retesting without cues.
\end{abstract}

A negative effect of part-set cuing occurs when some elements of a set of to-be-remembered material are made available in a test, with the result that the recall of the rest of the set of material is worse than recall without the part-set cues (Roediger, 1973). The three experiments reported in this article deal with the question of reversibility of this effect and, specifically, with the possibility that the effect vanishes when a second test is given without cues. Three studies have suggested that this is so (B. H. Basden, D. R. Basden, Church, \& Beaupre, 1991; D. R. Basden, B. H. Basden, \& Galloway, 1977; Roediger, Stellon, \& Tulving, 1977).

This apparent reversibility of the part-set cuing effect is interesting for two reasons. First, it appears to be unique in the annals of memory research: forgetting is first induced by an experimental operation, and shortly thereafter, memory is restored by withdrawal of that intervention. Second, such an effect seems important for contending theoretical accounts of the part-set cuing effect. For example D. R. Basden et al. (1977) and B. H. Basden et al. (1991) took their results to mean that part-set cuing effects are the result of disruption of editing-retrieval and not the result of a strength-competition process (Watkins, 1975).

The present experiments dealt with two questions about the reported reversibility of the part-set cuing effect. First, does it show up in the form of increased recall of new items on the second test? To date, researchers finding reversibility have reported only mean recall on successive tests. These scores, however, represent a mixture of intertest forgetting, intertest retention, and reminiscence (recall of items for the first time on the second test). The latter recall events are the most important for assessing reversibility. If some items are "inhibited" by cues on the first test, they should be "released" and recallable in a second test without cues.

The second question concerns the extent to which recovery from the effects of part-set cuing is more than just

Correspondence concerning this article should be sent to H. Park, Department of Psychology, University of Southern California, Los Angeles CA 90089. an instance of reminiscence. It is by now well known that in two successive recall attempts, the second will contain items not present in the first test, in cued as well as in free recall (Otani \& Hodge, 1991; Payne, 1987). The change in cuing conditions might be irrelevant if a general reminiscence effect were responsible for increased recall in the second test.

\section{EXPERIMENTS 1A-1B}

The first two experiments were essentially attempts to replicate the experiments by D. R. Basden et al. (1977) and B. H. Basden et al. (1991), but with the additional purpose of examining recall components across tests.

\section{Method}

Design. The experiment was a within-subject test of the part-set cuing effect. The subjects first studied a list of 10 eight-item semantic categories with blocked presentation. A series of category-name cued recall tests followed, in which five tests were administered without instance cues (NC) and five with four instance cues (IC).

Materials. Word lists were constructed from 12 categories; eight instances were selected from the Murdock (1976) norms. Two categories served as primacy and recency buffers. A microcomputer program constructed lists by dividing the 10 categories at random into two groups of 5 , one for tests without cues and one for tests with cues. In each category, four items were designated at random as the cues and four as recall targets. The sequence of conditions in the study list as well as the test sequence was created by successive random ordering of NC and IC category pairs. Additional counterbalancing occurred for categories against conditions and instances against cue-target status.

Procedure. The test sessions were conducted by a microcomputer. The subjects first read instructions that described the nature of the study list and that directed the subject to read each category name and instance carefully. The study trial consisted of 10 category presentations with the category name and eight instances appearing at 2-sec intervals. Immediately following the study phase, a screen of test instructions appeared that directed the subject to attempt recall of eight (NC tests) or four (IC tests) instances. In both cases, a category name appeared, followed by either eight question marks on separate lines (NC) or four category instances (IC) and four question marks. The instance cues were presented at the rate that was used during list presentation. The subjects were allowed $60 \mathrm{sec}$ for recall per category for both types of tests, and they responded by typing instances on the microcomputer keyboard.

The first test was followed immediately by a 5 -min series completion task and then by a second recall test that followed the procedure of the 
first test, except that in all tests only the category name was used as a cue. In the second test, the subjects had to attempt to recall the originally presented eight instances.

Experiments $1 \mathrm{~A}$ and $1 \mathrm{~B}$ followed the same procedures, the only difference between them being that the instructions in Experiment $1 \mathrm{~B}$ discouraged guessing in an attempt to reduce the intrusion rate observed in Experiment $1 \mathrm{~A}$.

Subjects. There were 24 (Experiment 1A) and 20 (Experiment 1B) undergraduate student subjects.

\section{Results and Discussion}

The top panel of Figure 1 shows the percentages of target instances recalled on the NC-NC and NC-IC tests in Experiments 1A and 1B. These data show first that there was a negative part-set cuing effect in the first test: target-set recall was better without cues in Experiment 1A $[t(23)=2.38, p<.026]$ and in Experiment $1 \mathrm{~B}[t(19)=$ $3.78, p<.001]$. In the second test, recall was still greater for NC-NC than for IC-NC, but the difference was smaller and not significant $(p>.05)$ in either experiment. As Figure 1 indicates, total recall dropped for the NC-NC tests and rose slightly for the IC-IC tests.

This pattern of outcomes resembles the results reported by D. R. Basden et al. (1977) and B. H. Basden et al. (1991). However, an analysis of the components of recall presents a different picture: the NC-NC tests and IC-NC tests did not differ in terms of recall of new or of "reminiscence" items in the second test. In Experiment $1 \mathrm{~A}$, the mean numbers of new items recalled were
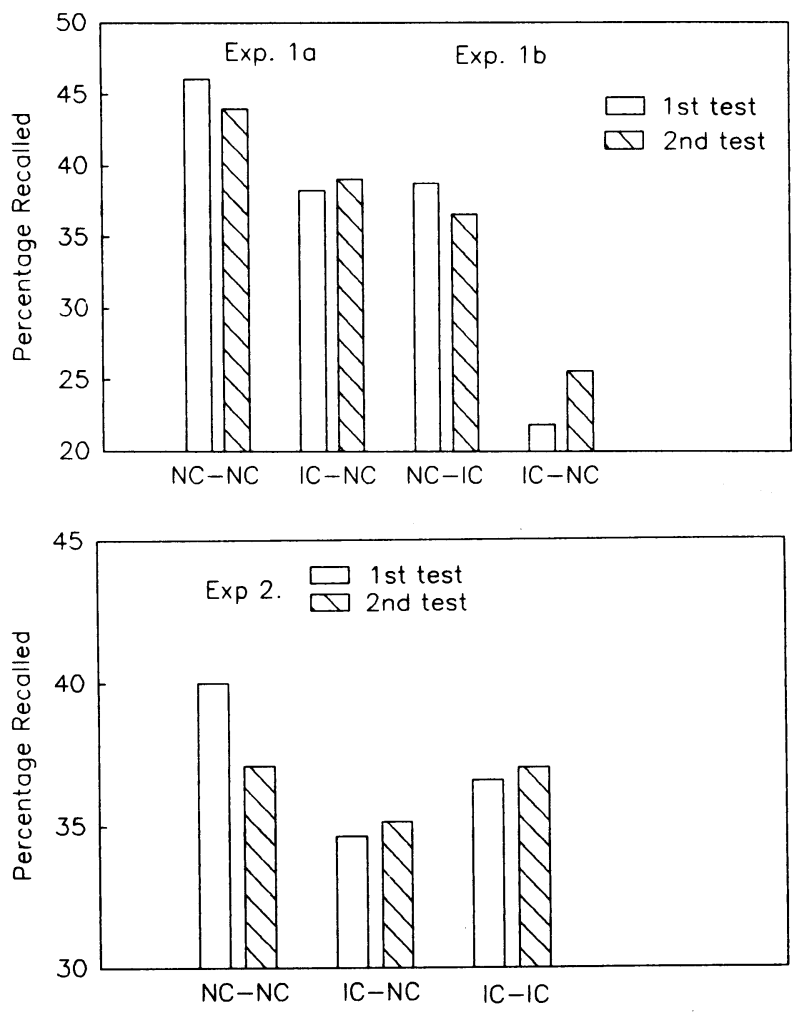

Figure 1. Percentage recall of target-set items in Experiments 1A-1B (upper panel) and Experiment 2 (lower panel).
0.54 and 0.92 for the NC-NC and NC-IC tests, corresponding to reminiscence rates of $6.3 \%$ and $9.1 \%$. In Experiment $1 \mathrm{~B}$, the corresponding figures were $0.90(7.1 \%)$ and $0.70(7.0 \%)$ for the NC-NC and NC-IC tests. Only the difference between raw reminiscence scores in Experiment 1A approached significance $(p=.08)$.

Unexpectedly, the two conditions did differ in intertest forgetting: more recalled items from the first test were forgotten between tests for NC-NC tests than for IC-NC tests. The means and conditionalized percentages were $0.87(11.8 \%)$ and $0.58(9.8 \%)$ for NC-NC and NC-IC in Experiment 1A, and $1.05(17.1 \%)$ and $0.30(8.6 \%)$ in Experiment 1B. Since reminiscence was greater than forgetting for the IC-NC tests, a hypermnesia effect was obtained in that condition. The reverse was true for NC-NC, producing a net loss across tests.

\section{EXPERIMENT 2}

The major purpose of Experiment 2 was to add a reminiscence control condition: two successive tests with the same part-set cues each time. To claim that the partset cuing effect is reversible by removing part-set cues, it must be shown that the number of new target instances in the second test is greater for an IC-NC test sequence than for an IC-IC test sequence.

\section{Method}

Design and Procedure. The experimental design for Experiment 2 was the same as that for Experiments $1 \mathrm{~A}$ and $1 \mathrm{~B}$, but with the addition of the IC-IC condition. Twelve categories were used, four for each of the NC-NC, IC-NC, and IC-IC test sequences. The categories now consisted of six rather than eight instances, with three-instance cue and target sets. The construction and presentation of lists and tests otherwise followed the procedures of Experiments $1 \mathrm{~A}$ and $1 \mathrm{~B}$, with test duration reduced to $45 \mathrm{sec}$ per category. In the IC-IC condition, the same instance cues were presented in the first and second tests.

Subjects. There were 60 undergraduate student subjects in Experiment 2.

\section{Results}

The bottom panel of Figure 1 shows recall of target instances in the first and second tests for each condition. A part-set cuing effect was again obtained in the first test: recall was greater for the NC-NC condition than in either the IC-NC or the IC-IC conditions combined [ $t(59)=$ $2.17, p<.05$ ]. (Unaccountably, first-test recall scores were somewhat greater for IC-IC tests than IC-NC tests.) The results for the second test are essentially the same as those in the first two experiments: total recall dropped slightly for the NC-NC tests and rose slightly for both the IC-NC and the IC-IC tests, with no reliable variation among the means $(F<1)$.

The components of recall in the second test also show a pattern much like that obtained in the first two experiments. First, there was no difference in recall of new items. The means and conditionalized percentages of new items recalled in the second test were $0.47(6.4 \%), 0.50$ $(6.3 \%)$, and $0.48(6.3 \%)$ for the NC-NC, IC-NC, and IC-IC conditions. Second-and replicating the results of 
the first two experiments-intertest forgetting was greater when the first test was uncued. The mean numbers and conditionalized percentages of items lost across tests were $0.8(17.0 \%)$ for NC-NC, $0.43(10.6 \%)$ for IC-NC, and $0.43(10.1 \%)$ for IC-IC tests.

\section{GENERAL DISCUSSION}

The main conclusion from this set of experiments is that the part-set cuing effect is not reversible, at least not through the simple experimental operation of retesting recall without part-set cues. The number of new items recalled on a second test without cues following an initial test with cues was no greater than the standard reminiscence effect obtained in two successive tests without part-set cues or in two successive tests with cues. Figure 2 summarizes this outcome in a different way. It shows the total number of unique items recalled over the entire two-test recall period for the NC-NC and IC-NC conditions of all three experiments. It is clear that the recall deficit existing at the end of the first test (at $60 \mathrm{sec}$ in Experiments $1 \mathrm{~A}$ and $1 \mathrm{~B}$ and $45 \mathrm{sec}$ in Experiment 2) was not made up in the second test period, and also that there were no strong signs of convergence of the cumulative recall curves over the course of the second test.
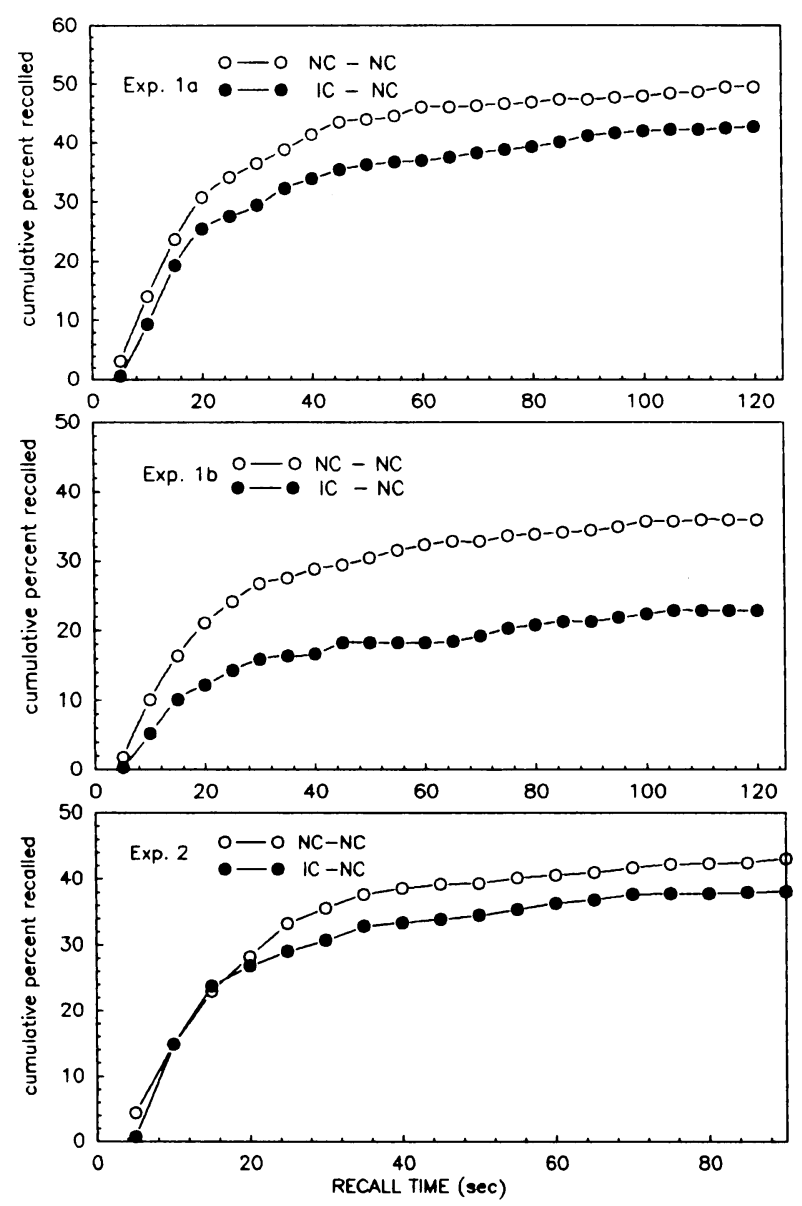

Figure 2. Cumulative recall of target items over both test periods in Experiments 1A, 1B, and 2.
All three experiments produced an unanticipated outcome: intertest forgetting was greater across two tests without part-set cues than across a first test with cues and a second test without cues or across two cued tests. Combined with small or nonexistent differences in reminiscence, this appears to underlie the interaction of cuing condition and test found in the present experiments and presumably in those in the literature. The difference in intertest forgetting itself may be nothing more than an item-selection effect if it is assumed that the target-set items recallable in the presence of part-set cues are stronger, and more resistant to interference, on the average, than target-set items recalled without cues.

How do the results bear on theoretical accounts of the part-set cuing effect? If "disinhibition" of target-set items in a second test without cues is required by the editing-retrieval hypothesis of part-set cuing effects, that hypothesis is facing difficulties. In some respects, the results seem to fit expectations derived from accounts that attribute the part-set cuing effect to strength-competition factors. In all three experiments, the material recalled best in the second test was the cue-set material presented in the first test: $53.1 \%$ in Experiment 1A, 78.7\% in Experiment $1 \mathrm{~B}$, and $52.2 \%$ in the IC-NC condition of Experiment 2. Clearly, these items had undergone a strengthening by virtue of their first-test presentation. Strength-competition theory would seem to require that target-set recall would be impeded in a later test by such items. It could be argued that the part-set cuing effect in the first test is in fact an editingretrieval disruption and that an independent process (list strength) works against increased target-set recall in a later recall attempt; but testing such a hypothesis appears difficult. Perhaps the best theoretical assessment of the present results would take the form of a simulation via SAM (Raaijmakers \& Shiffrin, 1981), that model having already been applied with some success to the part-set cuing effect as well as to recall over repeated tests.

Finally, the fact that the adverse effect of the initial part-set cue presentation persists through a second test without cues raises the question about how long such effects will last. This question is especially interesting in connection with part-set cuing effects that are obtained in the generation of instances from semantic memory (Sloman, 1991).

\section{REFERENCES}

Basden, B. H., Basden, D. R., Church, B. A., \& Beaupre, P. (1991). Bulletin of the Psychonomic Society, 29, 213-216.

BASDEN, D. R., BASDEN, B. H., \& Galloway, B. C. (1977). Inhibition with part-list cuing: Some tests of the item strength hypothesis. Journal of Experimental Psychology: Human Learning \& Memory, 3, 100-108.

MURDOCK, B. B. (1976). Item and order information in short-term serial memory. Joumal of Experimental Psychology: General, 105, 191-216.

Otani, H., \& Hodge, M. H. (1991). Does hypermnesia occur in recognition and cued recall? American Journal of Psychology, 104, 101-116.

PAYNE, D. G. (1987). Hypermnesia and reminiscence in recall: A historical and empirical review. Psychological Bulletin, 101, 5-27.

RAaijmakers, J. G., \& Shiffrin, R. M. (1981). Search of associative memory. Psychological Review, 88, 93-134.

RoEDIGER, H. L., III (1973). Inhibition in recall from cuing with recall targets. Journal of Verbal Learning \& Verbal Behavior, 12 , 644-657.

Roediger, H. L., III, Stellon, C., \& Tulving, E. (1977). Inhibition from part-list cues and rate of recall. Journal of Experimental Psychology: Human Learning \& Memory, 3, 174-188.

SLoman, S. A. (1991). Part-set cuing inhibition in category-instance and reason generation. Bulletin of the Psychonomic Society, 29, 136-138.

WATKINS, M. (1975). Inhibition in recall with extralist "cues." Journal of Verbal Learning and Verbal Behavior, 14, 294-303.

(Manuscript received April 2, 1993.) 